\title{
Design features of the orb web of the spider, Araneus diadematus
}

\author{
Alun ap Rhisiart \\ Fritz Vollrath \\ Department of Zoology, \\ University of Oxford, \\ Oxford OX1 3PS, UK \\ and \\ Zoologisches Institut, \\ Rheinsprung 9, CH 4051 \\ Basel, Switzcrland \\ and \\ Department of Zoology, \\ Universitetsparken B135,
} 8000 Aarhus, Denmark

Received 12 October 1992 First revision 8 June 1993 Second revision 27 July 1993 Accepted 27 July 1993

1045-2249/94/\$5.00

(C) 1994 International Society for Behavioral Ecology

Analysis of orb webs of the garden cross spider (Araneus diadematus) showed that these vertical webs have a significant up/down asymmetry. Experiments demonstrated that the spider runs down faster than up, and thus confers a relatively higher foraging value to sections below the hub. Simulations suggested that the density of capture spiral spacing, prey size, and the density of prey should all affect the capture efficiency of a web. Webs lose effective capture area because of overlap of the capture zone around each thread; the smaller the prey, the finer the mesh can be without losing effective area. Lower sectors of the web have a particular mesh size (height and length of capture spiral segments) throughout, whereas in the upper sectors the mesh size changes, widening from the hub towards the periphery. Key words: orb web, web asymmetry. [Behav Ecol 5:280-287 (1994)]

$\mathrm{T}$ he spider's web discharges a number of different functions such as catching prey, allowing the early detection of mates or enemies, and providing a shelter against climate or predators. Arguably its main function is that of a trap. This is not always fully appreciated and in this article we aim to to supply some new arguments in favor of this basic assumption. We start with the premise that the architecture of the web reflects its builder's adaptive response to its prey-insects of various sizes, shapes, and weights.

Nature furnishes us with a large variety of web types, including simple tangles or sheets, complicated tangle-sheet combinations, more or less geometric orbs, and finally, just threads, sticky or nonsticky (Stowe, 1986). The easiest to study for its adaptive geometry is the orb web in its simplest form, the 2-dimensional, point-symmetrical array of nonsticky radii overlaid with a sticky spiral thread. We shall argue that the geometric symmetry of this construction is largely shaped by its function as a filter of aerial "plankton," mainly flying insects. By filter we do not assume a purely passive role for the web. Every web carries an active ingredient, the spider, which in most cases must deploy rapidly to prevent a prey escaping from the sticky threads that trapped it in the first place (Eisner et al., 1964).

Following Vollrath (1992) we view the spider's orb web as incorporating a trinity of foraging decisions. First, the spider makes decisions as to the timing and locality of its web: when to build it, where to place it, how to orient it, and when to give up and go elsewhere. This is considered site choice (Janetos, 1986). Second, the spider constructs its web and makes decisions about the allocation and the distribution of silk in the structure. This is considered a search path (Vollrath, 1992) because the web results from the spider's searching for prey (albeit not interactively but in vacuo). Third, the spider, which monitors its orb from the hub, cannot respond equally quickly to prey at all points of its web, and a web's trapping value declines to- ward the periphery. This is considered central place foraging (Vollrath, 1992) because the spider uses the hub as the center of its prey capture activities.

Viewing the orb as a structure that represents three different, though partly interrelated aspects of foraging behavior (site choice, search path, and central place foraging), we can identify separate selection pressures for each of the three adaptive responses. Once identified, we can study these hypothetical selection pressures, either in computer simulations or experiments, or even in field observations. We shall, in this article, restrict ourselves to the investigation of web geometry (i.e., search path and central place foraging) and leave out decisions on site choice. Moreover, we shall concentrate on exploring promising avenues for continued research, rather than the elucidation of all aspects of one particular example.

\section{Orb shape and up-down asymmetry}

Orbs of the common garden spider (Araneus diadematus) are usually built in the vertical and show an obvious asymmetry between the area above and below the hub (Vollrath and Mohren, 1985; Witt et al., 1968). We analyzed the geometry of orb webs (Figure la) built in the laboratory under controlled conditions to study the variability of this asymmetry with a view toward identifying a possible adaptive function of this asymmetry in trapping prey.

\section{Material and methods}

Araneus diadematus were caught as small immatures in two localities and kept in the laboratory under standard conditions (16:8 L:D, $50 \% \mathrm{RH}, 25^{\circ} \mathrm{C}$ ambient temperature) in standard frames (Plexiglas $30 \times 30 \times 5 \mathrm{~cm}$ ). Webs were photographed backlit against a dark background. The resulting negatives were projected to life size onto a digitizing tablet (Houston Hipad) and digitized with a resolution of $0.1 \mathrm{~mm}$ using our own software (Digitizer v1.2). The web coordinates were then analyzed following 
traditional parameters (Witt and Reed, 1965) but using our own software (SPIDERMAC II v1.8).

\section{Results and discussion: web asymmetry}

Our analysis confirmed that vertical webs of $A$. diadematus are asymmetrical. The radials of the south (the quadrant facing down) were significantly longer than those of the north (the quadrant facing up), east and west (the quadrants between) were intermediate and did not differ from each other at the $1 \%$ level (Figure 1; Table 1). The south also had more radials than the east and west, which in turn had more than the north (Figure 1), and the south had a more evenly spaced capture spiral (termed mesh size) than the north, east, or west. The south also has a greater number of spiral threads than the north, east, or west (Figure 1) because in the south the spider often incorporates U-turns into this path, thus weaving to and fro.

We hypothesize that the more even mesh of the south is not a side effect of the spider's web-building algorithm caused by the closer spacing of the radials in this web section. On the contrary, we hypothesize that this regular spacing is an adaptation of the spider that has evolved to put a higher investment in the more profitable part of the web.

Can we test this hypothesis? In the following we shall demonstrate (1) that the shape of the web with the longer south reflects the spider's hunting behavior as a central place forager; (2) that in simulations the spacing of the mesh has a great effect on a web's efficiency as a trap; and (3) that the more even mesh of the south means that we must amend the theory of Peters $(1947,1951,1954)$, that the capture spiral follows a particular rule of construction (the segment rule).

\section{Probability of capturing trapped prey}

But why should the south be treated differently by the building spider? The spider can run down (153.9 $\mathrm{mm} / \mathrm{s}$ ) faster than up $(86.9 \mathrm{~mm} / \mathrm{s}$ ) (calculated from Masters and Moffat, 1983) and locate prey faster in the direction in which it faces (Klärner and Barth, 1982; Weissmann, 1987). These observations suggest that different sections of the web have different value to the spider, which sits at the hub and has to catch a prey insect that hits the web before it can escape. It seems beneficial for the spider to face down and combine the two features (orientation and gravity) into one great advantage over the prey.
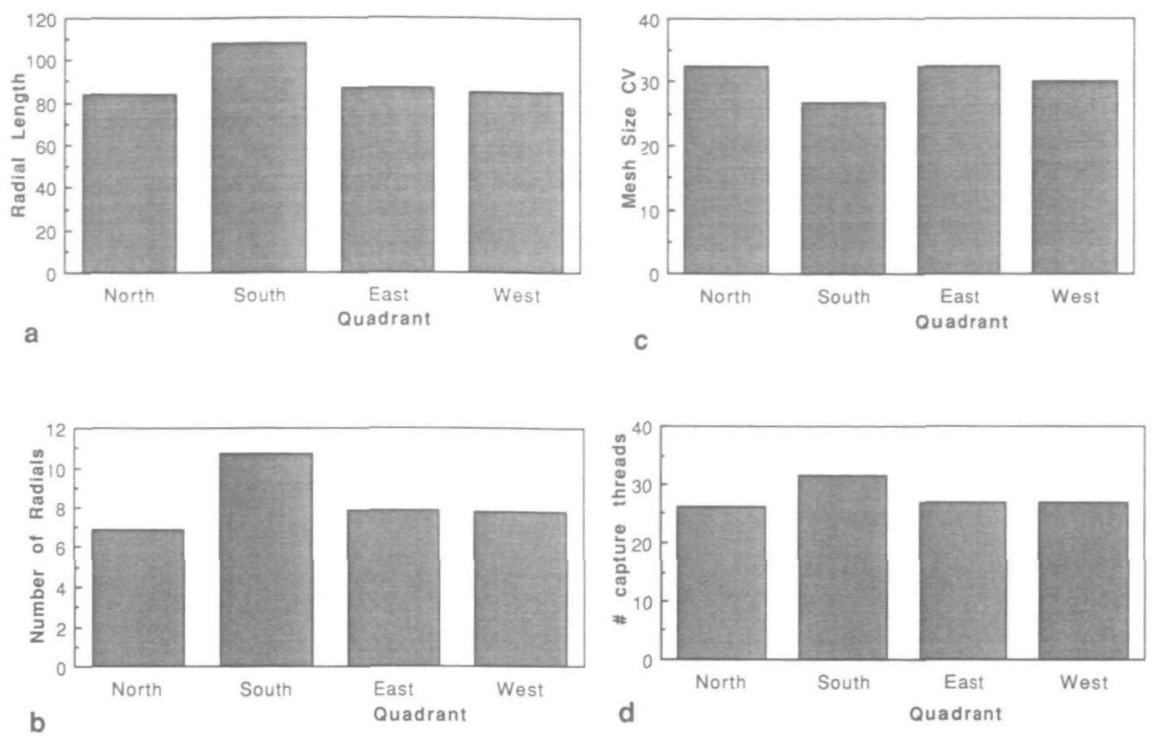

We are treating the spider's situation as a central place foraging task. Prey are more likely to escape the farther from the center they are; this is a simple consequence of increasing the spider's travel time. The spider also has to detect the presence of the prey before it can act, and the farther out it is, the weaker the signal passed through the radii (Masters et al., 1986). In an experiment we tested the time it took $A$. diadematus to reach various parts of its web from its resting place at the hub of the web. The speed at which a spider can reach different parts of a web could be affected by (1) gravity (in vertical webs), and (2) differences in web geometry (e.g., the number of radii in north and south). We therefore carried out further experiments to determine which was more important.

\section{Methods}

The spiders were allowed to build webs in Perspex frames, $30 \mathrm{~cm}$ square and $5 \mathrm{~cm}$ deep. All webs were built in the vertical position. We then carefully tossed small specimens (length $\approx 10 \mathrm{~mm}$ ) of locusts (Locusta migratoria) into the webs with tweezers, one per web. The spiders' reactions were recorded on a Sony Video 8 camcorder $(25 \mathrm{fps}$ ) for later analysis on a Sony EV-S800.

Playing back a spider's response frame by frame, we measured the angle of the prey relative to the center, the initial distance between spider and prey, and the time the spider needed to reach the prey.
Figure 1

Characteristics of Araneus diadematus webs. Typical orb web of $A$. diadematus. The radials in the south as opposed to the other quadrants: (a) were longer, (b) there were more of them, (c) the mesh was more even, and (d) there were more spiral threads.

Table 1

Characteristics of Araneus diadematus webs

\begin{tabular}{lcrl} 
Quadrant & Number of radii & Radial lengths/mm & Capture threads \\
\hline North & $6.8 \pm 0.1(194)$ & $83.8 \pm 1.4(194)$ & $26.1 \pm 0.6(189)$ \\
West & $7.7 \pm 0.1(194)$ & $84.9 \pm 1.6(194)$ & $27.0 \pm 0.7(192)$ \\
East & $7.9 \pm 0.11(194)$ & $84.8 \pm 1.6(194)$ & $27.0 \pm 0.6(194)$ \\
South & $10.7 \pm 0.16(194)$ & $107.7 \pm 2.1(194)$ & $31.5 \pm 0.8(192)$ \\
\hline
\end{tabular}

Number radii of $A$. diadematus webs in each quadrant are significantly different $\left(F_{3,772}=199.881^{* * *}\right.$ ANOVA), as are radial lengths $\left(F_{3,772}=45.028^{* * *}\right.$ ANOVA $)$ and number of capture threads $\left(F_{3,763}=13.097^{* * *}\right.$ ANOVA $)$. The south has more radii than the other three quadrants, and east and west have more than the north (Games-Howell statistic). The south also has longer radii with more spiral threads than the other three, but there is no difference between north, east and west (Games-Howell statistic). All figures are mean $\pm \mathrm{SE}(n)$. 
Table 2

Reaction times and orientation times of Araneus spiders in vertical webs

\begin{tabular}{lllc} 
Quadrant & $\begin{array}{l}\text { Reaction time } \\
(\mathrm{s})\end{array}$ & $\begin{array}{l}\text { Orientation time } \\
(\mathrm{s})\end{array}$ & $\begin{array}{l}\text { Mean speed } \\
(\mathrm{mm} / \mathrm{s})\end{array}$ \\
\hline North & $0.07 \pm 0.02(8)$ & $0.14 \pm 0.02(12)$ & $88.4 \pm 16.0(9)$ \\
West & $0.07 \pm 0.2(9)$ & $0.15 \pm 0.04(10)$ & $92.8 \pm 12.0(13)$ \\
East & $0.08 \pm 0.03(10)$ & $0.14 \pm 0.03(11)$ & $115.2 \pm 16.9(16)$ \\
South & $0.41 \pm 0.36(10)$ & $0.49 \pm 0.4(10)$ & $164.4 \pm 14.5(13)$ \\
\hline
\end{tabular}

Neither reaction time $\left(F_{3,33}=0.76\right.$ ANOVA) nor orientation time $\left(F_{3,33}=0.784\right.$ ANOVA) was significant, but Araneus traveled much faster south than in any other direction $\left(F_{9,47}=4.85, p<.01\right.$ ANOVA). All figures are mean $\pm \operatorname{SE}(n)$.

We measured the distance of the spider's front leg to the nearest part of the prey (not hub to prey). The initial reaction from the spider was to orient toward the prey and move 10 or $20 \mathrm{~mm}$ out from the center in its direction, and then pause with the first pair of legs on the radii leading out to the prey. When the prey moved again, the spider would run out rapidly to the prey and attack.

Differences in travel times between various parts of the web may have been due to differences in local structure: for example spiral and radial spacing varies between north and south. Therefore we repeated (as a control) these experiments using webs that were built in the vertical but which we then laid in the horizontal. We then dropped prey into them exactly as described above.

We divided the web into eight octants and averaged the speeds at which the spiders traveled to catch prey placed within an octant. These speeds were then multiplied by 10 and 20 to calculate the distance that a spider could travel in that many time units. Results from the east and west of the web were pooled to smooth irregularities resulting from sample size and random factors as east and west are in all respects equivalent. The results of our analysis of travel time are displayed as a contour map.

As a further test of the gravity versus web geometry hypotheses, we used webs that had all been built normally in the vertical plane. "Prey" in this experiment was standardized by touching a radial thread in the lower part of the web with a thin piece of stiff wire attached to one prong of a tuning fork $(392 \mathrm{~Hz})$. In a paired-sample test, each web was tested twice, once in its normal position, once upside down. Gravity was therefore constant, but the web geometry was that of the north in half the

Figure 2

The distances that $A$. diademalus could reach in 10 and 20 time units in a typical web. Distances were determined by dropping prey into typical vertical webs and examining filmed prey capture sequences frame by frame (25 fps). Figures are speeds $(\mathrm{m} / \mathrm{s})$.

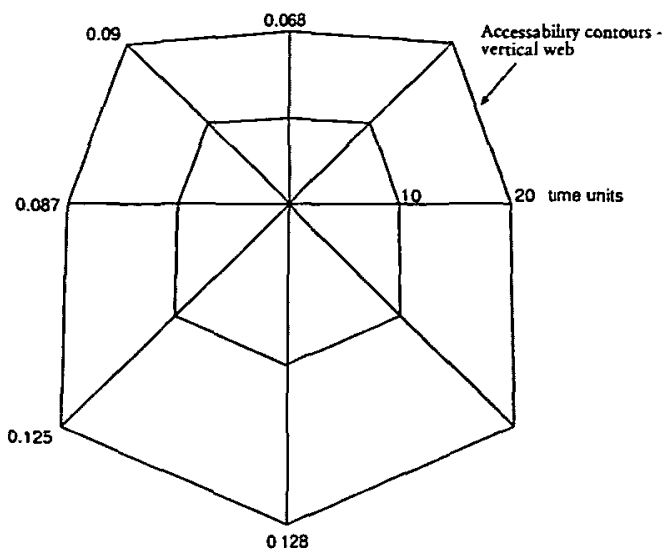

cases, and that of the south in the other half. The order of presentation (normal or upside down) was alternated. A total of 12 webs were tested in this way and filmed as above.

\section{Results}

The time that a spider took to reach the prey can be broken into three periods: reaction time, orientation time, and travel time (Klärner and Barth, 1982). Both reaction time and orientation time were longer for prey that landed in the south of the web, but not significantly so (see Table 2). However, the spiders traveled much faster downward than in any other direction (Table 2). The reaction and orientation times were only a small fraction of the total time elapsed from the prey hitting the web and the spider reaching it. The spiders did not run faster toward the south in the horizontal webs, even though this architecture was the same $\left(F_{2,6}=1.71\right)$. Indeed, the trend was in the other direction [north: $191 \pm 75.6$ (3), south: $60 \pm 4.7$ (2)], suggesting that the difference between running up and running down was due to gravity rather than web geometry.

As further confirmation of this, there was no significant difference between normal and upsidedown webs in the third experiment $\left(F_{1.11}=0.134\right.$ ANOVA). The mean speed running south was 126.9 $\pm 21.78 \mathrm{~mm} / \mathrm{s}$ (mean $\pm \mathrm{SD}$ ) for control webs and $131.8 \mathrm{~mm} / \mathrm{s}$ for upside-down webs.

\section{Descussion}

The plot of travel times as a contour map clearly matched the shape of an $A$. diadematus web (Figure 2). This strongly suggested that webs were built in such a way as to take account of the profitability of different sections of the finished structure. It suggested that radial lengths were not chosen to fit the size of the frame, but were chosen to extend to distances that can be reached within a certain time. Considering the capture spiral as a search path, we might also say that the spider invested energy (silk) in each part according to the expected return. Thus, peripheral sections were comparatively less valuable in terms of supplying food, even though they received as many hits as more central sections (closer to the hub and the waiting spider), because a greater proportion of these hits may escape before the spider could reach them.

The speeds obtained in the upside-down experiment were rather lower than in the earlier ones for south (though still considerably higher than the figures obtained earlier for north). The most likely explanation for this is simply that the distance traveled was slightly underestimated in this experiment, 
a result of not having a prey on screen to which we could measure the distance. The first figures (Table 2) correspond very well with Masters and Moffat (1983), and are probably more reliable. The underestimation applied to both normal and upside-down webs, of course.

As the distance from the hub increased, the chances of snared prey escaping increased more rapidly in the north than in the south because of differences in up and down running speeds. This would apply even if the web was built symmetrically, as the difference is due to gravity. Given this, we may expect silk investment in the web to decrease (i.e., the mesh to become coarser) in proportion to its distance from the hub; and that decrease would be most marked in the north. This assumes that there is some connection between mesh size (i.e., capture spiral spacing) and the probability of a prey being captured. We examined this idea in a simulation of prey capture by computer generated orb "webs."

\section{Simulations of the web as a filter}

One of the costs of building a web is the metabolic cost of the silk, and spiders appear to be limited in the amount of sticky capture silk available at any one time (Eberhard, 1988). Other important costs are the construction behavior both in terms of exposure time and metabolism (Lubin, 1986; Witt et al., 1968). Thus any web spider is faced with the choice of whether to build a high-cost web with many lengths of silk, or a low-cost one; and, for a given amount of silk, whether to build a large web with a coarse mesh, or a smaller and finer-meshed onc. The most cost efficient web will depend critically on the size distribution of prey that passes through the space occupied by a web and the density of these prey items. There has been some controversy over whether an orb web functions as a filter, i.e., over the importance of mesh size for prey capture. For example, some data suggest (albeit not strongly) that mesh size may be irrelevant (Nentwig, 1983), whereas theoretical considerations suggest that it may be important (Eberhard, 1986). To clarify this, we simulated the interaction of prey (circles of varying diameter) and webs (networks of lines in various shapes).

\section{Methods}

We used computer simulations to study changes in optimal mesh and web size resulting from changes in these parameters. Our simulated webs were regular and symmetrical with equally spaced spirals. The program, written in SMALLTALK V/Mac on an Apple Macintosh computer, produces the required number of equally spaced radii overlaid by evenly meshed spirals of the required pitch. Different mesh sizes and number of radii could be chosen, and new nodes were added until either the area or the spiral length reached the specified value. In this way, it was possible to produce webs that had different mesh sizes and equal spiral lengths, or different mesh sizes and equal overall areas (Figure 3a-d). The program then simulated a specified number of prey of a given size passing through the web space and a surrounding area and counted the number of prey that hit the spiral (not the radii). Prey positions were chosen from random
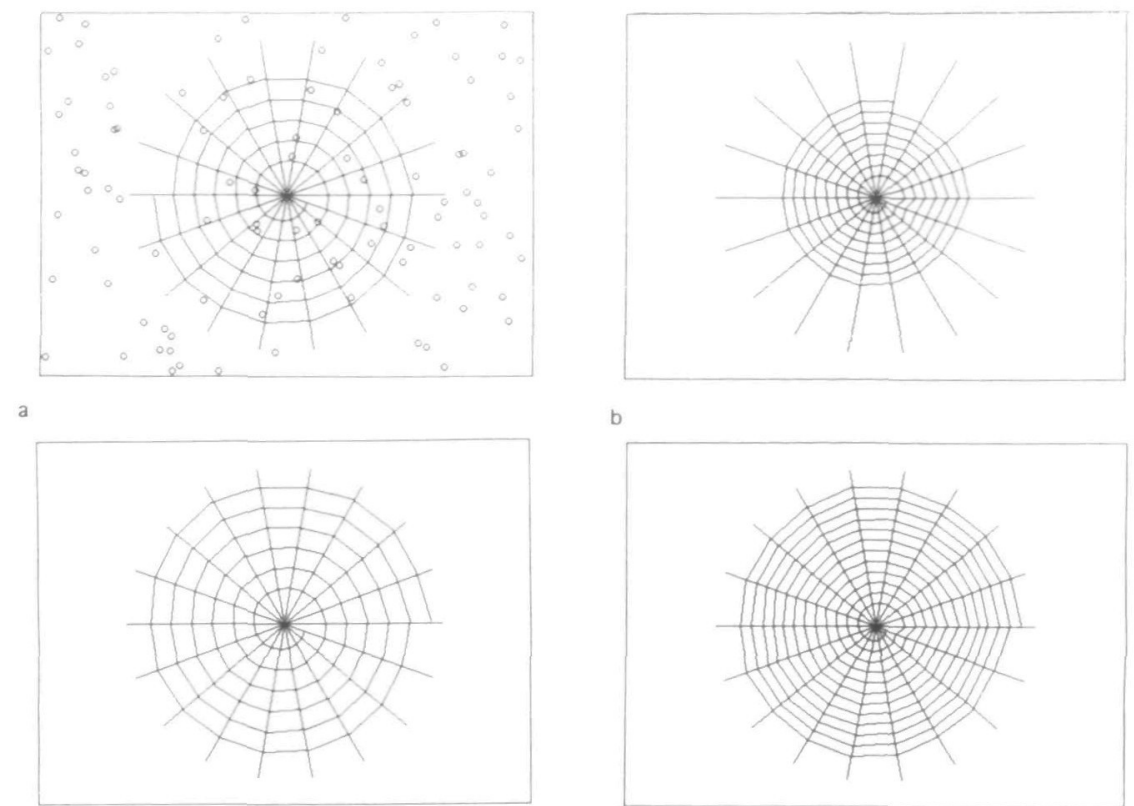

b

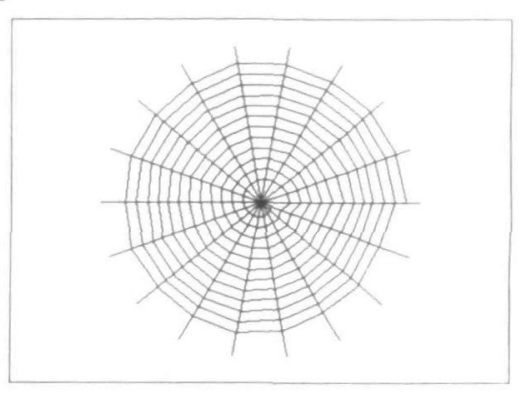

numbers constrained to fit within the total prey space, which was larger than the web. The spiral was drawn onto a bitmap, and at each prey location, and within prey-size pixels either side of that location, the program checked to see if the pixel was black (i.e., part of a spiral); if so, it counted a hit. Our program could also draw spirals according to a different rule-keeping the successive angles the same, or could simulate prey flying into digitized images of real webs.

\section{Prey size}

For the first set of simulations, we compared the efficiency of two webs with different mesh sizes ( 10 and $20 \mathrm{~mm}$ ) and varied the prey diameter from $60 \%$ to $240 \%$ of the smaller mesh size (i.e., $2 \mathrm{~mm}$ to $24 \mathrm{~mm}$ ). Our first two simulation series take account of the costs of silk and building behavior by constraining the webs to have equal overall spiral lengths, and were run for two different prey densities: high density (400) and low density (50).

The amount of silk available is one important constraint on the sort of web a spider can build (Eberhard, 1988; Witt et al., 1968). Another is given by the dimensions of the frame in which the web has to be fitted. Given a fixed area, would a spider do better to fill that area with a fine-meshed, more costly web, or with a coarse-meshed, cheaper web? Our second simulation series examined this by using simulated webs in which the overall area was kept the same, and again we compared webs in high density (400) and low density (50) environments.

The number of prey chosen to represent rich and poor environments in these simulations is fairly arbitrary, as reliable measurements of prey density in the vicinity of webs were not available. The prey densities may appear to be high; note that this was to represent the total number of prey passing through or near the web over a day or longer.

The results are shown in Figure $4 \mathrm{a}$ for webs with equal spiral lengths and a rich environment $(400$ prey within or near to the web). For a given web
Figure 3

Simulated webs and their prey. Four webs produced by the simulation program. (a) shows prey circles. $(a, b)$ have equal spiral length. (c, d) have equal area. Mesh sizes are 20 $\mathrm{mm}(\mathrm{a}, \mathrm{c})$ and $10 \mathrm{~mm}(\mathrm{~b}, \mathrm{~d})$. The black rectangle represents the area within which the prey were confined. 


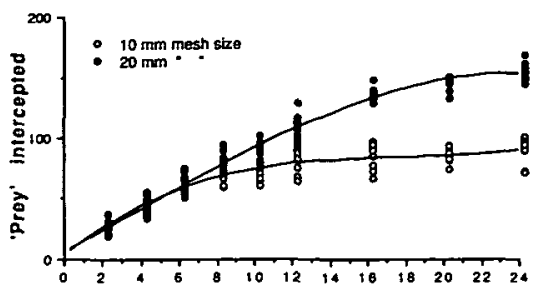

a

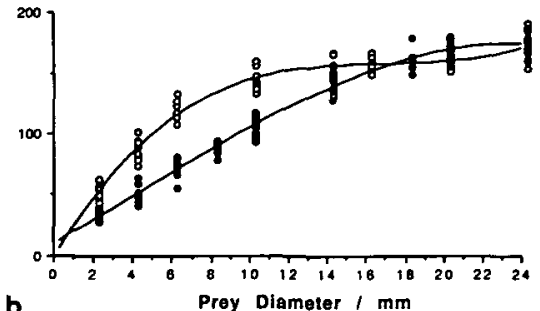

Figure 4

Simulated prey capture by simulated orb webs. Plots of various diameter "prey" caught by two model "webs" with mesh spacings of $10 \mathrm{~mm}$ (open circles) and $20 \mathrm{~mm}$ (filled circles). The ordinates represent the number of prey hitting a strand. $(a, b)$ the effect of differences in prey diameter (prey density 400); (c, d) the effect of differences in prey density of the environment (prey size $6 \mathrm{~mm}$ ) on the capture rate of the two types of spacings. Webs in a and $c$ had equal spiral lengths but different areas; in $b$ and $d$ the areas are equal but the finer meshed spiral was twice as long. Prey encounter rates were simulated in the computer by imposing disks onto line drawings representing stylized orb webs with 18 radials and evenly spaced capture spirals. Disks that hit a strand of web were counted once. Each environment contained 400 prey placed at random and al simulations were repeated ten times. The lines represent 3 rd order regressions.

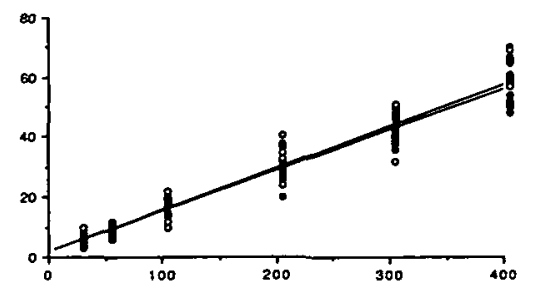

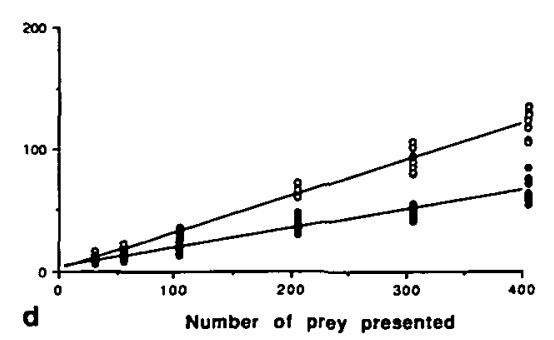

c

size, it appeared that the number of prey caught increased with prey size until the size of the prey exceeded the mesh size, and then the number caught reached an asymptote. When prey were smaller than the mesh size, large- and small-meshed webs were equally good, but large-meshed webs (which had a larger area for a given length of silk) caught more larger prey, and thus did better overall. However, these differences appeared only when the environment was quite rich; at low prey densities mesh size was not important in either a web with equal spiral length or equal area.

The results of a simulation in which web area was kept constant are shown for rich environments in Figure $4 \mathrm{~b}$. As in the previous case, the number of prey caught was correlated with prey diameter until this equaled the mesh size, but this time the asymptotes were equal. Thus, finer meshed webs appeared to be better traps, and we will show that this was because of their longer spiral lengths.

\section{Prey density}

In a further simulation series, prey size was kept constant but we investigated the effect of prey density in greater detail. Again, the efficiency of two webs $(10 \mathrm{~mm}$ and $20 \mathrm{~mm}$ constant mesh size) was compared in each simulation. The number of prey presented was $25,50,100,200,300$, and 400 within the total area described above. In a way similar to the prey size experiment, there were four simulation runs: (1) two webs of equal spiral lengths and a constant prey size of $6 \mathrm{~mm}$; (2) equal spiral lengths and a prey size of $20 \mathrm{~mm}$; (3) webs of equal area and 6-mm prey; and (4) equal area and 20mm prey.

The results of our simulation for prey that were smaller than the mesh size (see Figure $4 \mathrm{c}$ for $6-\mathrm{mm}$ prey) show that there was no difference in the numbers of prey captured at any prey density in webs that had the same spiral length. At the larger prey size, or with webs that had equal area (Figure 4d), there was no difference at low prey densities, but as prey density increased the advantage of the finer meshed webs over the coarser meshed ones steadily increased.

To summarize: there was no difference in numbers of prey captured by coarse- and fine-meshed webs when areas were kept equal if prey were large, or when spiral length was kept equal if prey were small. For small prey and equal area fine $>$ coarse; for large prey and equal spiral length, the opposite is true-coarse $>$ fine.

\section{A model for thread placement efficiency}

The picture so far is somewhat confusing: mesh may be important (at high prey density) or it may not (at low prey density). When it was important, coarse webs were better (if spiral length was equal) or worse (if area was equal) than fine webs. Our interpretation of these apparent contradictions makes use of a simple mathematical model. For the first scenario, we assumed that the prey, if it touches a single thread, will be held long enough for the spider to reach it. For the second scenario, we considered the situation where the prey would escape too quickly unless it was more securely held by two or more threads.

Let mesh size be $m$, and the radius of a prey approaching the web $r$. On passing through the web, the prey will touch one of the spiral threads if it approaches to within $r$. Therefore, if $m \leq 2 r$, the efficiency of the web will be $100 \%$ and all prey flying through the web will touch a spiral thread; if $m>2 r$ some of the prey will fly through. The capture area is therefore the strip of space within $r$ on either side of the sticky spiral, and the prey capture area (a) is

$$
\mathrm{a}=\mathrm{L} \cdot 2 r
$$

where $L$ is the length of the sticky spiral. This strip of space could be in a straight line or it could be kinked (refer to Figure 5), but this would make no difference to the capture area for a given length of silk as long as the threads do not overlap, and are at least $2 r$ apart: if the line is kinked (as at nodes), the capture area lost on the inside is gained on the outside, and so the total area remains constant. This also means that the number of radii is irrelevant to the calculation of capture area, although it is important for other reasons (e.g., to provide a framework). However, if one part of the spiral thread were to cross over another part there would be a loss of capture area, because of overlapping areas.

Prey do not remain stuck indefinitely in webs, however, and a spider has to reach a prey quickly. The shortest way from one point to a set of random points in its vicinity is a "star" of lines, but the shortest way of connecting these points is a spiral (Krieger, 1992). Thus the most efficient way of minimizing the mean distance from the spider to a prey that could hit anywhere within the total web area is to build its capture thread in a spiral, overlaying a star of stiff radials. This spiral has a minimum mesh size determined by the prey size $(r)$, and a maximum length determined by structural and travel time constraints. In this situation the mesh size is important, but the spiral does not act as a filter (we will return to this point later).

The spiral covered area within which prey are not within touching distance of a thread (Figure $5 b)$ is

$$
\mathbf{b}=(m \cdot \mathrm{L})-(\mathrm{L} \cdot 2 r)
$$

and the web efficiency $(c)$ is

$$
c=L \cdot 2 r / m \cdot L=a /(a+b) .
$$


This scenario is the one used in the simulations, and we can now explain the results of our simulations. The number of prey captured is correlated with prey size, $r$, because there is a resultant increase in the capture area $2 \mathrm{Lr}$. This will reach an asymptote when the prey diameter equals the mesh size because after that the capture areas of adjacent spiral threads overlap and are wasted (Figure 5).

Keeping spiral length constant, a larger mesh size is better for catching large prey (increasing area or decreasing cost for the same area), but both are good at catching small prey (Figure 4a). When prey are larger than the mesh size, there is waste of capture areas of adjacent threads. For prey smaller than the small mesh size, both webs (fine and coarse) have equal capture efficiencies. If the area is kept constant, as would be the case where the spider has to build within a restrictive frame, then a smaller mesh size would mean that there is a longer spiral thread, and therefore a larger capture area, until the prey is larger than the mesh. Therefore, decreasing the mesh size in this situation is profitable only if most of the prey encountered are small. However, it would be a waste of silk (and building time) if most of the prey are large relative to the mesh size (Figure $4 \mathrm{~b}$ ).

All this assumes that a prey needs to touch one thread only, rather than two or more. For small prey (e.g., Drosophila spp.) this is probably true, but larger prey might break through, and so the web might have to be built in such a way that the prey touches more than one thread as it passes through. In this situation, mesh size is important, and the web can be said to act as a filter. An optimal mesh size would then be a compromise between one that is large enough not to waste capture area for small prey (that do not need to touch two spiral threads), and one that is small enough to ensure that larger prey do touch two. With this in mind, it should be possible to examine the escape abilities of prey of different size classes and discover empirically where the optimum lies. Of course, even a single strand might hold a heavy prey (with plenty of kinetic energy) briefly, and thus by diverting its course bring it into contact with additional strands below. We have evidence from high speed filming (1000 fps) that a single strand of a medium sized $A$. diadematus web can in this way result in the capture of a bumble bee in full flight.

\section{Peters's segment rule revisited}

We showed that sectors below the hub (the south) were significantly more evenly meshed than sectors above the hub (the north) (Figure 1). This observation appears to conflict with conclusions in seminal articles on spider web-building behavior (Peters, 1939, 1954). As the fact of a functional northsouth difference is important for our central argument we used our digitizing set-up to repeat $\mathrm{Pe}$ ters's measurements with a higher resolution than was available to him.

Using ruler and compass, Peters measured various distances on web photographs of Araneus diadematus, among others the mesh of the capture spiral. By mesh is meant the length and height of the trapezoid segments that are formed where the capture spiral intersects with radii-like rungs on a ladder (Figure 6A). Although Peters's data showed

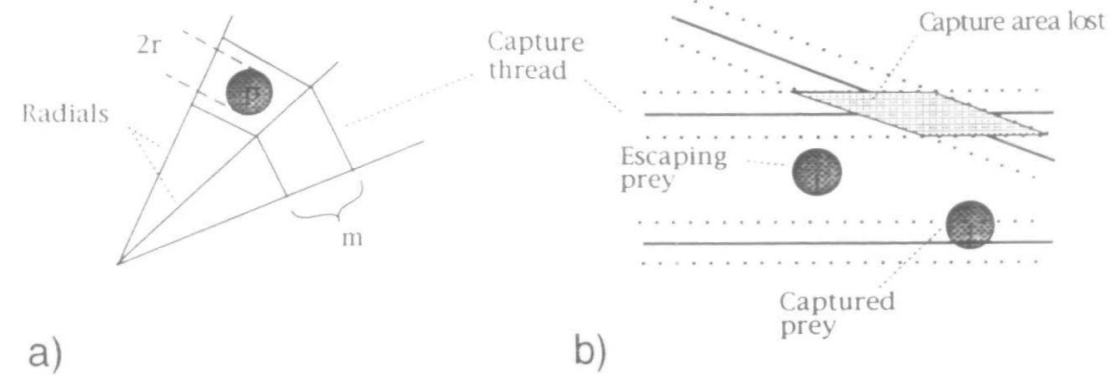

Figure 5

much scatter and did not fully convince him, he deduced that, on balance, it was suggestive of a certain rule, which he termed the segment rule. The segment rule claims that the spider when laying down spiral strives to maintain for each mesh trapeze a fixed ratio of length to height. Peters acknowledged that there seem to be a number of exceptions to this rule, one possibly occurring in the south.

We, too, found a wide scatter if we lumped our data from the four web quadrants. However, when we separated our data by quadrant, we found that there was a difference between the south and the other three quadrants in this regard. In the south the spider kept the mesh fairly even throughout all but the peripheral two or three segments. Almost all webs showed a highly significant correlation between mesh height (along the radii) and width (between the radii) in the north, east and west, but not in the south. An ANOVA of the 14 webs, with web ID as a factor, mesh width as the regressor, and mesh height as the dependent variable, we found that a regression of mesh height on mesh width was highly significant (north: $F_{1,312}=$ $161.057^{* * *}$; south: $F_{1,461}=1.870$ ). Moreover, the gradient in the north was much higher than the south, where it was not at all pronounced (north: mesh height $=0.106 \times$ width $+1.43 ;$ south: mesh height $=0.011 \times$ width +2.86 ). To achieve this up/down asymmetry in mesh size the spider incorporates reverses (by changing direction during spiral construction, sec Figure 1) into southern sections of the spiral.

It may be argued that the difference we found in the south of the web has nothing to do with its position in relation to gravity, but instead is due to differences in web geometry in that sector. In particular, there tend to be more, and more closely spaced, radii in the south than in other sectors. Our hypothesis does not reject the idea that such

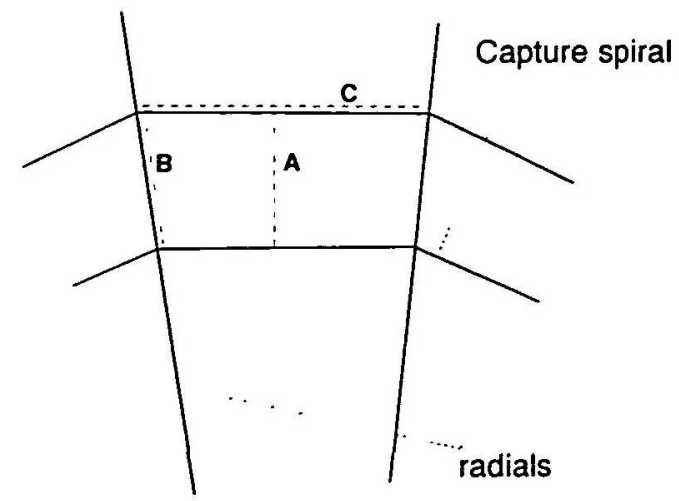

A simple model to describe the capture area of a web. (a) Prey of diameter $2 r$ passes through a web. (b) If it approaches a thread closer than $r$, it is intercepted. The grey area is the amount lost when two threads cross.
Figure 6

The dimensions measured by Peters and the present authors to determine a correlation between mesh size along and between radii. Peters measured the correlation between $\mathrm{A}$ and $\mathrm{C}$, giving rise to the "segment rule." We measured the correlation between $\mathrm{B}$ and $\mathrm{C}$, as it is simpler, and is the measure that the spider uses during building. 
geometric factors may be important, so long as the result is a finer, more evenly-meshed capture spiral in the south. To test which factors are really im. portant, we allowed Araneus to start building webs in our normal setup, and then turned the frames upside down after the radii were built. Now, the radial wheel in the south has a geometry typical of the north, and vice versa. The result was as before: a significant relation between mesh width and height in the north $\left(F_{1,178}=208.072^{* * *}\right)$ but not in the south $\left(F_{1,167}=2.687, p=.103\right)$. The gradients became a little farther apart than usual (north: mesh height $=0.289 \times$ width +0.633 ; south: mesh height $=0.024 \times$ width +1.856 ).

The hypothetical segment rule could be envisioned as one of a set of decision rules that the spider uses to determine the placement of threads. It is, indeed, possible to use such rules in an artificial web-building algorithm, and asymmetric orbs can readily be simulated in the computer if different weightings are used for the lower and the upper half of the web (Gotts and Vollrath, 1991; Vollrath, 1992). Thus it is well possible that the up/down asymmetry of the Araneus diadematus orb web is an adaptive response incorporated into the spider's inherited set of web-building rules.

\section{GENERAL DISCUSSION}

A spider makes three types of foraging decisions. First, there is the choice of a site, which affects all the remaining decisions. Second, there is the construction of the web; the radials and frame threads provide a micro-environment, and the laying down of the capture spiral can then be considered a search path, with the encounters occurring after the path has been traversed, rather than during it, as is more normal. Finally, the spider sits at the center of the web (or in some species in a hiding place near the web but linked to its hub by a signal line) and dashes out to catch entangled prey, a central place foraging task (Pyke, 1984).

The relationship between the architecture of the web (its form), and its efficiency at catching prey (its function), is direct but not quite straightforward. The size of the prey and the encounter rate make a big difference to this relationship, as does the number of capture threads needed to hold the prey until the spider can reach it (Eberhard, 1986). Some spiders do not attempt to attack some of the prey, but wait until they dismantle the web and then ingest the prey together with the web (Pasquet and Leborgne, 1990). The experiments with travel times confirm that the shape of Araneus webs is in large part determined by the central foraging aspect. Where this does not apply the web could presumably assume other, more circular, geometries. It is noteworthy that Cyclosa spp., for example, build very regular, circular, albeit vertical webs (ap Rhisiart $A$ and Vollrath $F$, unpublished observations), and one might look for an explanation in its prey catching strategies. It is obvious why species with horizontal webs should build circular ones, as indeed they do (Eberhard, 1987).

The broad shape of the web can be predicted from travel times to the various quadrants, but what about the fine structure? We could perhaps predict differences between webs specialized for catching larger prey (which could escape) and small prey. Those for small prey would be circular, and finely meshed because with small prey the capture area either side of each spiral turn is narrow. The mesh can therefore be made finer without losing capture area to overlap. If we knew how long prey were held on average (and what common or preferred prey are), we could examine the distance to the outer mesh, and work out the time required to reach a point there and the probability of prey escaping in that time. This would give us the "pursuit threshold" in this system, i.e., the probability of capture below which it is not worthwhile for the predator to pursue a prey. In most predator-prey systems the threshold depends on the cost of pursuit; in spiders, it would be the cost of laying down silk in unprofitable areas.

We were initially interested in the effect of mesh regularity, because under some circumstances (e.g., if the spider is drugged or sprayed with pesticide, or disturbed during building) the web can be less regular than normal (Witt et al., 1968; Samu and Vollrath, 1992). Our model shows that regularity is important largely to the extent that spiral threads avoid crossing over each other. If more than one thread is required to hold prey fast, then an irregularly spaced web will differ in its efficiency at catching different size classes of prey in different parts. To some extent this may be advantageous, and because spiral thread has an associated cost, the searching part of the foraging strategy can be adapted according to the expected return from different parts of the web. For example, as the distance from the web hub increases, the profitability will decrease because a greater proportion of the prey will escape before the spider can reach it. The spider may therefore decrease its investment in these areas by laying down a larger mesh size. This is what happens, particularly in the north, where the gradient of access times increases more steeply. We confirm Peters's (1951) observations that there is a relationship between mesh height and mesh width in Araneus webs; a relationship, however, that does not apply (or perhaps is much weaker) in the southern sectors of the web. Our results show, however, that this is due to the spider actually treating the south differently during capture spiral construction, and not just because of differences in radial geometry.

North and south differ not only in thread geometry but also in thread material. The capture threads of $A$. diadematus are covered with a liquid coat of hygroscopic compounds mixed with glucoprotein glue (Vollrath et al., 1990; Vollrath and Tillinghast, 1991). The initially uniform coat quickly forms droplets that are crucial to the proper function of the capture threads (Vollrath and Edmonds, 1989) and their stickiness (Edmonds and Vollrath, 1992). A comparison of droplet sizes shows that those of the south are significantly larger than those of the north (Edmonds and Vollrath, 1992), a difference that is not due to a downward flow of the coating. On the contrary, it is likely that this difference is due to differential coating by the spider, which exudes more liquid glue in the south. This, too, might contribute to a higher capture effectiveness in the south, and it may be another indication that Araneus treats the web in the south differently during construction. 
We thank Dieter Ebert for suggestions, Monika Müller for help in droplet measurements, and Paul Watson and another anonymous referee for helpful critical remarks.

\section{REFERENCES}

Eberhard WG, 1986. Effects of orb-web geometry on prey interception and retention. In: Spiders: webs, behavior and evolution (Shear WA, ed). Stanford, California: Stanford University Press; 70-100.

Eberhard WG, 1987. Effects of gravity on temporary spiral construction by Leucauge mariana (Araneae: Araneidae). J Ethol 5:29-36.

Eberhard WG, 1988. Behavioural flexibility in orb web construction: effects of supplies in different silk glands and spider size and weight. J Arachnol 16:295-302.

Edmonds D, Vollrath F, 1992. The contribution of atmospheric water vapour to the formation and efficiency of a spider's web. Proc R Soc Lond B 248:145-148.

Eisner T, Alsop R, Ettershank G, 1964. Adhesiveness of spider silk. Science (NY) 146:1058-1061.

Gotts NM, Vollrath F, 1991. Artificial intelligence modelling of web-building in the garden cross spider. J Theor Biol 152:485-511.

Janetos A, 1986. Web site selection: are we asking the right questions? In: Spiders: webs, behavior and evolution (Shear WA, ed). Stanford, California: Stanford University Press; 9-22.

Klärner D, Barth FG, 1982. Vibratory signals and prey capture in orb-weaving spiders (Zygzella $x$-notata, Nephila clavipes; Araneidae). J Comp Physiol 148:445-455.

Krieger M, 1992. Radienbau im Netz der Radnetzspinne. (Diplom Arbeit.) Basel: Universität Basel.

Lubin YD, 1986. Web building and prey capture in the Uloboridae. In: Spiders: webs, behavior and evolution (Shear WA, ed). Stanford, California: Stanford University Press; 132-171.

Masters W, Markl H, Moffat A, 1986. Transmission of vibration in a spider's web. In: Spiders: webs, behavior and evolution (Shear WA, ed). Stanford, California: Stanford University Press; 49-69.

Masters M, Moffat A, 1983. A functional explanation of top-bottom asymmetry in vertical orb webs. Anim Behav 31:1043-1046.

Nentwig $W, 1983$. The non-filter function of orb webs in spiders. Oecologia 58:418-420.
Pasquet A, Leborgne R, 1990. Prey capture efficiency and prey selection from insects intercepted by trap in four orb-weaving spider species. Acta Oecol 11:513523.

Peters HM, 1939. Über das Kreuzspinnennetz und seine Probleme. 27:777-786.

Peters HM, 1947. Zur Geometrie des Spinnenetzes. Z Naturforsh 2b:227-232.

Peters HM, 1951. Untersuchungen über die Proportionierung im Spinnen-Netz. Z Naturforsch 6b:90-107.

Peters HM, 1954. Worauf beruht die Ordnung im Spinnen-Netz? Umschau 54:368-370.

Pyke G, 1984. Optimal foraging theory: a critical review. Annu Rev Ecol Syst 15:523-575.

Samu F, Vollrath F, 1992. Spider orb webs as bioassays for agrochemicals. Entomologia Exp Appl 62:119-126.

Stowe MK, 1986. Prey specialization in the Araneidae. In: Spiders: webs, behavior and evolution (Shear WA ed). Stanford, California: Stanford University Press; 101-131.

Vollrath F, 1992. Analysis and interpretation of orb spider exploration and web-building behavior. Adv Study Anim Behav 21:147-199.

Vollrath F, Edmonds D, 1989. Modulation of normal spider silk by coating with water. Nature (Lond) 340 : 305-307.

Vollrath F, Fairbrother WJ, Williams RJP, Tillinghast EK, Bernstein DT, Gallagher KS, Townley MA, 1990. Compounds in the droplets of the orb spider's viscid spiral. Nature (Lond) 345:526-528.

Vollrath F, Mohren W, 1985. Spiral geometry of the garden spider's orb web. Naturwissenschaften 72:666-667.

Vollrath F, Tillinghast EK, 1991. Glycoprotein glue inside a spider web's aqueous coat. Naturwissenschaften 78 : 557-559.

Weissmann $M, 1987$. Web-building and prey capture in two orb weavers (MSc thesis). Oxford: Oxford University.

Witt PN, Reed CF, 1965. Spider web-building: measurement of web geometry identifies components in a complex invertebrate behavior pattern. Sciences (NY) 149: 1190-1197.

Witt PN, Reed CF, Peakall DB, 1968. A spider's web: problems in regulatory biology. Heidelberg: Springer. 